\title{
EL LEGADO DE SCHUMPETER AL ESTUDIO DE LA ADMINISTRACIÓN DE EMPRESAS*
}

\author{
Sergio A. Berumen \\ Real Academia de Ciencias Morales y Políticas \\ Madrid, España \\ lee_berumen@racmyp.es
}

\begin{abstract}
Resumen
En este artículo se expone cómo los planteamientos más sólidos del pensamiento neoschumpeteriano pueden ser de suma utilidad para el análisis de la administración de empresas. La teoría schumpeteriana se centra en el uso de conceptos de la Biología para analizar, por analogía, el comportamiento de las instituciones y los sistemas sociales. Según este enfoque, las señales que las empresas reciben del sistema económico son de tres tipos: a) las oportunidades tecnológicas y los beneficios esperados asociados a ellas, b) el comportamiento actual y esperado de la demanda por sus productos y por otros productos y c) los cambios en costos, precios, cantidades y rentabilidades en los mercados donde operan y en algunos otros.
\end{abstract}

Palabras clave: pensamiento neoschumpeteriano, administración de empresas, organizaciones.

\begin{abstract}
In this article it is stated that the most demonstrated statements of the neoschumpeterian theory might be extremely useful for the analysis of business administration. Schumpeterian theory focuses in the use of analogies or biology concepts to analyze the behavior of institutions and social systems. For this focus, the signals that companies receive from the economical system are of three types: a) the technological opportunities and the benefits expected, which are closely related to them, $b$ ) the current behavior and the expected one from the demand from its products and other products and c) the changes in costs, prices, quantities, and rentabilities in the markets in which they operate, and in some others.
\end{abstract}

Key words: neoschumpeterian theory, business administration, organizations.

* El presente artículo fue realizado en el seno del grupo de investigación Competitividad y Desarrollo Local en la Economía Global, que es auspiciado por la Comisión Europea y la Fundación Grupo Santander. Agradecemos la revisión y los valiosos comentarios que han hecho a este trabajo Fabio Bagnasco Petrelli (Universidad de Padua), Luis Miguel Jiménez Herrero (Universidad Complutense de Madrid) y Petra Schoenghen (Universidad Libre de Berlín). 


\section{Introducción}

Según los autores neoschumpeterianos, las ciencias sociales se estructuran en función de sistemas dinámicos, complejos e interdependientes que se despliegan en un tiempo histórico e irreversible. Para estos economistas, los agentes deben tomar decisiones cuyo impacto sólo se evidencia luego de un tiempo considerable, en contextos de incertidumbre e interacciones holísticas entre los agentes y las variables agregadas (véase Silverberg, et al., 1988). La visión global, holística y macroscópica intenta establecer por qué el conjunto es más que la suma de las partes y de explicar las relaciones dinámicas de las partes en el todo, del todo en las partes y del todo en las totalidades. Como lo señala Jiménez Herrero (1996: 275 y sig.), en este contexto es importante interesarse en la relación que existe entre el hombre y la naturaleza en su complejidad, totalidad y dinámica propia, utilizando el microscopio como un nuevo instrumental científico de observación del sistema global.

El pensamiento neoschumpeteriano se nutre fundamentalmente de cinco canales teóricos básicos: a) el invalorable legado de Joseph A. Schumpeter, b) la Escuela Evolucionista de Economía, c) el pensamiento poskeynesiano, d) muy recientemente, la Escuela Conductista y e) las contribuciones formuladas en el seno de la Escuela Neoclásica. En términos generales, los autores neoschumpeterianos se enfocan en el uso de conceptos de la Biología para analizar, por analogía, el comportamiento de las instituciones y los sistemas sociales. Durante el siglo XIX algunos autores de la Escuela Histórica Alemana, como Roscher (1843 y 1894) y Schmoller (1900), hicieron constantes referencias a los aspectos biológicos al analizar las relaciones entre las empresas, los sistemas económicos y el entorno subyacente a estos. Argumentaron que las teorías de la evolución histórica se desarrollan en escenarios de cambio cíclico, por lo cual la economía no es una ciencia normativa ni, desde luego, exacta.

A inicios del siglo XX, Veblen (1919) intentó desarrollar una teoría socioeconómica de la evolución basada en los principios darwinianos de herencia, variación y selección. Consideró los instintos, las convenciones, los hábitos y las instituciones como las unidades de herencia, mutación y selección en las sociedades humanas. De acuerdo con sus planteamientos, la evolución de las sociedades y las organizaciones implicaba la modificación de las preferencias individuales conforme la sociedad adquiría nuevos conocimientos o desarrollaba nuevas ideas y conceptos. Para este autor, los individuos, las organizaciones y el entorno social estaban en un continuo cambio motivado por su interacción. Veblen, a diferencia de Marx, pensaba que la evolución era indeterminada, pues sufría múltiples forcejeos, movimientos laterales y retrocesos, por lo que estaba, finalmente, sujeta a decisiones azarosas y de la cambiante voluntad humana.

Marshall (1890) reconoció la necesidad de que la economía explorara el uso de los principios de la teoría de la evolución biológica (por ejemplo, admitió el carácter estático de los conceptos extraídos de la mecánica clásica). Su análisis resalta los cambios irreversibles de la actividad económica, sugiriendo la presencia de un tiempo real distinto del tiempo mecanicista, propio de la economía neoclásica. Para él, las mismas condiciones en momentos distintos no producen los mismos resultados $\mathrm{y}$, además, muy probablemente nunca se vuelven a dar esas mismas condiciones. Un aspecto 
destacable en su obra es el proceso de ajuste de las organizaciones a largo plazo, pues si bien no hay una referencia explícita a la metáfora biológica, este concepto ha sido utilizado posteriormente por los neoschumpeterianos para explicar el proceso de selección en la sociedad.

Los trabajos de Marshall y Veblen se complementaron con las investigaciones de Schumpeter. Este economista de origen austriaco definió la evolución en términos de los cambios que ocurren en el seno de las organizaciones; colocó el cambio tecnológico en el centro de la evolución, y al empresario como el ente innovador de las empresas. Intentó, a nuestro parecer inútilmente, hacer compatible el concepto del equilibrio general walrasiano con la evolución ${ }^{1}$. A partir de este momento, el pensamiento schumpeteriano y la economía neoclásica se volvieron muy difícilmente reconciliables (salvo en algunas excepciones muy específicas).

De acuerdo con el enfoque schumpeteriano, son los empresarios y/o los consejos de administración los encargados de modificar el orden de las cosas: en su afán por incrementar ganancias, cambian rutinas, y al hacerlo, crean nuevas trayectorias de evolución, para las cuales hay que crear nuevos hábitos y nuevas instituciones. En el caso de las grandes empresas, las decisiones

1. El equilibrio general establecido por Leon Walras es el eje de la teoría neoclásica contemporánea, el cual se basa en la consideración de que es plenamente alcanzable un equilibrio único y estable en el largo plazo, en el que conviven la racionalidad de los agentes económicos y la interdependencia entre mercados, y en donde todo es igual a la suma de las partes. Hay constancia de la admiración que en sus inicios Schumpeter le prodigaba a Walras; de hecho, en 1910 publicó un trabajo titulado Marie Esprit Leon Walras. se asumen por los consejos de administración u otros cuerpos colegiados, los cuales son los responsables de generar nuevas trayectorias de evolución. La noción del empresario como tomador único e infalible de decisiones es un enfoque obsoleto que no tiene aplicación en el seno de las grandes empresas, asunto que en su día Schumpeter no fue capaz de prever.

A partir de los trabajos de Friedman (1953), el enfoque neoclásico y sus versiones derivadas inverosímilmente consideraron que la evolución ocurre en un ambiente estático (y con una función y objetivo atemporal o inmutable en el tiempo), en el cual no existe interdependencia alguna entre el entorno y los agentes que operan en él y que todo tiende a un equilibrio estable e inmutable debido al surgimiento de un orden natural espontáneo. De acuerdo con este enfoque, los animales y las plantas se comportan como si intentaran maximizar las posibilidades de supervivencia de su material genético al enfrentar unas condiciones determinadas. Contrario a lo que han escrito autores de la Escuela de Economía de Chicago, Schumpeter (1911) consideró que una organización que se rige por parámetros capitalistas nunca deja de moverse, por lo cual es imposible estudiarla a partir del postulado de estacionalidad. La búsqueda de utilidades y la acumulación de capital conducen a un aumento en el crecimiento. El impulso fundamental que alimenta dicho crecimiento proviene de los nuevos bienes consumibles, los nuevos métodos de producción, los nuevos mercados y las nuevas formas de organización de los individuos. El proceso de cambio es cualitativo, pero también cuantitativo. Prueba de ello es que las viejas industrias constantemente son reducidas en su alcance, e incluso algunas han desaparecido y han dado paso a otras nuevas. El resultado no ha sido únicamente 
una expansión cuantitativa de las organizaciones, sino también un cambio cualitativo en las estructuras. La creación de nuevas empresas y la desaparición de las viejas es para Schumpeter la destrucción creativa.

\section{El legado de Schumpeter al estudio de la administración de empresas}

La destrucción creativa schumpeteriana se refiere a que para crear algo nuevo es necesario destruir parte, gran parte o la totalidad de lo viejo. De acuerdo con este enfoque, la historia de la humanidad puede clasificarse en dos tipos de etapas: a) las de cambio incremental y b) las de cambio de paradigma tecnoeconómico. En las primeras, se ha mejorado lo existente hasta llevarlo casi al límite de sus capacidades; una vez que ha estado muy cerca del límite, las deficiencias de la tecnología, los sistemas organizacionales y el sistema socioeconómico se han vuelto patentes y por ello ha iniciado una frenética búsqueda para identificar y evaluar caminos alternativos de evolución. Las segundas se refieren a una nueva concepción para enfrentar los problemas y a los métodos para solucionarlos. Esto significa que conforme aparecen distintas alternativas, comienzan a disputar entre sí por cuál será la que se convertirá en la guía de la siguiente fase de cambio incremental. Cuál alternativa resulte vencedora dependerá de los grupos de apoyo que tenga cada una de ellas; se elegirá como camino de evolución básico la opción que: a) satisfaga ciertas condiciones, principalmente, la de ser capaz de superar las dificultades encontradas con la elección anterior y b) reúna el grupo de apoyo más fuerte.

Cuando estas condiciones tienen lugar en una sola industria o región se habla de un cambio de trayectoria tecnológica. Cuando el cambio se da en toda la sociedad, se trata de un cambio de paradigma tecnoeconómico (ver Freeman y Pérez, 1988). En ambos casos ocurre una modificación de los problemas que se consideran relevantes y de las que se cree son las formas correctas de solucionarlos. Esto es, las trayectorias tecnológicas y los paradigmas tecnoeconómicos simultáneamente son programas de investigación y desarrollo, y modelos o patrones de conducta a seguir: $j$ si bien se podrán atacar otros problemas, buscar otras alternativas de solución o adoptar otros programas de investigación, estos siempre estarán al margen de la política principal! Al darse un cambio de paradigma o de trayectoria, algunos o varios de los conocimientos, rutinas e instituciones desarrollados bajo el paradigma anterior se vuelven obsoletos por ser contrarios a las prácticas que ha impuesto la nueva trayectoria o paradigma para poder ser instrumentado. A este reemplazo de conocimiento, rutinas e instituciones es a lo que Schumpeter se refería con la original denominación de destrucción creativa.

Por ende, la destrucción creativa se refiere a una forma de competencia sustancialmente distinta a la que se pudiera encontrar en la teoría neoclásica. Lo que Schumpeter (1911) propone es un proceso que relega al equilibrio puramente competitivo. En virtud de lo anterior, la amenaza real para una organización está constituida por la introducción de un nuevo producto o una nueva tecnología por un competidor; nuevos negocios con frecuentes innovaciones harán desaparecer a los antiguos, y las nuevas empresas con nuevos productos o tecnología tratarán de obtener posiciones monopolísticas. Las innovaciones en los productos y la tecnología en la elaboración de estos, los mercados y los métodos de 
organización se suceden en forma de olas. Los empresarios son atraídos hacia el uso de tecnología de punta y los productos más innovadores; a la postre, estos aspectos se convierten en el motor del crecimiento económico que genera una expansión de los mercados. Durante esta fase de acelerado crecimiento económico, el capital (por ejemplo, los empresarios y/o los consejos de administración) son atraídos hacia los motores de crecimiento e ignoran otras variantes o posibilidades. A menudo, los nuevos empresarios y/o consejos de administración inician nuevas tecnologías y crean empresas que sirven para colocar productos en nuevos nichos del mercado o emular a los mismos pioneros.

La formulación de varias alternativas de evolución que superen las deficiencias de la línea de evolución seguida hasta el momento es un proceso que requiere de un periodo de entre cinco y quince años. El proceso de selección de la nueva alternativa dominante (nueva trayectoria tecnológica o nuevo paradigma tecnoeconómico) toma otra década. Por tanto, entre la detección de los límites del crecimiento de un paradigma o trayectoria y su sustitución por otra mejor adaptada a las nuevas condiciones transcurren entre diez y veinticinco años.

Ha sido una constante que cuanto mayor haya sido el éxito del paradigma o trayectoria anterior y cuanto más radical sea el cambio propuesto, mayor tiempo tardará en darse la transición. La destrucción creativa de Schumpeter sugiere que la industria tiene patrones de ciclos de vida de desarrollo caracterizados por un rápido crecimiento inicial y una reducción subsiguiente de ese proceso $\mathrm{y}$, en algunos casos, un declarado declive. Una industria crece rápido hasta que llega a saturar el mercado, una vez culminado este periodo, por lo que el crecimiento de la demanda del producto se relaciona con el ingreso y el crecimiento de la población en la medida en que crezca la competencia con nuevos productos y nuevas tecnologías que entren al mercado y sean capaces de atraer a los consumidores.

La propuesta general de Schumpeter resalta que el sistema económico se mueve empujado por los nuevos bienes de consumo, los nuevos métodos de producción y de transporte de las mercancías, los nuevos mercados y las nuevas formas de organización industrial que generan las empresas. Schumpeter señaló la diferencia entre el simple mejoramiento de tecnología y el movimiento lateral de procesos nuevos; para él, con el paso del tiempo un sistema que utilice sus recursos óptimamente podría resultar inferior a otro sistema que no se desempeñe tan óptimamente pero que sea más innovador. Sin embargo, el economista austriaco no fue capaz de identificar que se puede innovar sin necesariamente destruir. La supervivencia es posible (incluso si se continúan siguiendo los viejos cánones con los que se ha procedido) si se muestra adaptabilidad al cambio. Prueba de ello es el género humano: la humanidad, antes que desaparecer, ha sido capaz de cambiar y de adaptarse mediante formas que no están al alcance de otros mamíferos.

La semilla conceptual que en su día creó Schumpeter fue retomada posteriormente por otros economistas. En la década del setenta algunos autores revivieron el interés por incorporar al conjunto de herramientas del análisis económico el uso del análisis de la evolución biológica. El autor pionero de este enfoque fue Georgescu-Roegen (1967 en adelante). Sus principales aportes se centraron en las implicaciones de la Ley de la Entropía en el análisis de las organi- 
zaciones, las nociones sobre la escasez absoluta y la integración de conceptos de la termodinámica en la economía. En 1971 este autor publicó su The Entropy Law and the Economic Process (La ley de la entropía y el proceso económico) y años más tarde salió a la luz su Energy and Economic Myths: Institutional and Analytical Economic Essays (Mitos en torno a la energía y la economía: ensayo del análisis económico e institucional, de 1976). Para Georgescu-Roegen (1978), el crecimiento de las organizaciones se caracteriza por redistribuciones cuantitativas dentro de un espectro de bienes cualitativamente constantes. Esta apreciación se fundamenta en los principios termodinámicos, en virtud de que desde la óptica de las ciencias físicas no es posible la creación de materia y, por tanto, el proceso de producción en realidad es un proceso de transformación de la materia a través de la manipulación de la mano del hombre (si bien hay una degradación energética, la cual se sustenta en el principio de la entropía). En consecuencia, el crecimiento de las empresas es un proceso que conduce a la producción de bienes nuevos, en los que implícitamente hay una homogeneidad cualitativa; de ahí que el proceso económico se caracterice por generar redistribuciones cualitativas dentro de un ámbito de bienes cualitativa y cuantitativamente cambiantes.

Otro autor de notable importancia fue Daly (1968 en adelante), quien se dio a la tarea de fusionar conceptos de la Biología y la Ecología en el análisis económico, tales como el de capacidad de carga. Para este profesor, en su The Economics of The Steady-State (La economía en el estado estacionario, de 1974), el crecimiento económico es el aumento natural de tamaño por medio de la adición de material a través de la asimilación, mientras que el desarrollo económico es la expansión de las posibilidades con las que se cuenta y, de ese modo, el acceder a un estado superior y mejor. En el estricto sentido de las competencias de las empresas, el proceso de evolución económica es una manera particular de evolución, en el que concurren elementos como la organización económica, las mercancías (en tanto que son el insumo que dinamiza el interés de los consumidores), las tecnologías, los productores y el resto de los agentes económicos. En 1973 Nelson y Winter publicaron Analysis of the Corporation (Análisis de la empresa); en 1975 se publicó Sociobiology: The New Synthesis (Sociobiología: una nueva síntesis), de Wilson, y por último, en 1982 Nelson y Winter publicaron su influyente libro An Evolutionary Theory of Economic Change (Teoría evolucionista del cambio económico), en el que argumentaban que las interacciones entre los individuos, las empresas y su entorno regresan al centro del cambio económico, tal y como en su día lo explicaron Veblen y Schumpeter.

\section{El pensamiento neoschumpeteriano contemporáneo}

A partir del trabajo del Nelson y Winter ha habido un resurgimiento del interés sobre este enfoque y los temas que este considera relevantes. Entre los trabajos realizados en las décadas del ochenta y noventa pueden mencionarse los siguientes: Clark y Juma, Long-Run Economics (Economía del largo plazo); Loasby, Equilibrium and Evolution (Equilibrio y evolución); Verspagen, Uneven Growth Between Interdependent Economies (Crecimiento desigual entre economías interdependientes); Becker, Altruism, Egoism, and Genetic Fitness: Economics and Sociobiology (Altruismo, egoísmo y puesta en forma de la genética: economía 
y sociobiología) (si bien, como se dijo anteriormente, este autor es un destacado exponente del enfoque neoclásico); Hodgson, Economics and Biology (Economía y Biología) y, más recientemente, The Evolution of Institucional Economics: Agency, Structure and Darwinism in American Institutionalism (La evolución de las instituciones económicas: agencia, estructura y darwinismo en el institucionalismo estadounidense); England, Evolutionary Concepts in Contemporary Economics (Conceptos evolucionistas en la economía contemporánea); Vromen, Economic Evolution (Evolución económica); Freeman y Soete, The Economics of Industrial In-novation (La economía de la innovación industrial); Dosi et al., su multicitado Technical Change and Economic Theory (Cambio tecnológico y teoría económica); Kenneth Boulding, Evolutionary Economics (Economía evolucionista) y su original propuesta teórica sobre la idea del balance de materiales (derivada de los principios físicos), como el concepto spaceship-earth, en el cual se concibe a la tierra como una nave espacial, o proceso cerrado; y Dennett, Freedom Evolves (Evolución de las libertades).

Particularmente se recomienda el trabajo de Nelson y Winter (1982) y las contribuciones de Leydesdorff (a partir de principios de los años setenta). Para estos autores, los elementos que caracterizan a la teoría evolucionista se distinguen por: a) el foco de atención se centra en una variable (o un grupo de ellas) que cambia con el tiempo, y es de sumo interés el entendimiento del proceso dinámico que está detrás del cambio observado, y b) la variable o sistema en cuestión sufre variaciones parcialmente azarosas y existen mecanismos de selección sistemática entre esas variaciones. El cúmulo de las teorías planteadas por los autores neoschumpeterianos señala que en todo proceso de evolución se encuentran los siguientes aspectos: a) el replicador evolutivo o mecanismo de transmisión, b) el principio de variación y c) el mecanismo de selección. Afirman que, si bien pueden existir algunos otros errores de frecuencia mínima que ocasionen una interacción positiva, el resultado final es que el portador del gen estará dotado de una ventaja evolutiva y, en función de ella, los organismos son capaces de sobrevivir y de transmitir las características genéticas a sus descendientes. Este es el principio de la selección natural que caracteriza a la evolución darwiniana. En este tipo de evolución los genes son los mecanismos de transmisión. El principio de variación es el error de copia (el mecanismo de selección es aleatorio) proveniente de una mutación, y que produce cambios graduales sucesivos.

La función ambiental es definida como la capacidad del proceso natural y sus componentes de proveer bienes y servicios que satisfagan las necesidades humanas, directa o indirectamente ${ }^{2}$. Para estos pensadores, los grupos en los cuales se clasifican las funciones ambientales son: a) las funciones de regulación de los procesos ecológicos, b) las funciones de carga, principalmente la provisión de espacio físico y c) las funciones de producción de recursos materiales y energéticos. Estas funciones son insustituibles, y no es correcto que se dé una en perjuicio de otra: el criterio fundamental es que todas las funciones son un bien económico escaso. Por tanto, el flujo entre energía y materia dentro del sistema económico es concebido como un proceso

2. En este tenor, conviene advertir que la entropía termodinámica es una magnitud que mide la parte de la energía que no puede utilizarse para producir un trabajo. 
relativamente lineal y unidireccional, no circular; considera que la energía y la materia fluyen del medio ambiente al sistema económico y generan un desperdicio de materia y calor que fluye del sistema económico al medio ambiente. El flujo comienza con el agotamiento de la energía y los recursos materiales y termina con la contaminación del medio ambiente con material de desperdicio y calor.

Considerando estos principios, el flujo de materia en la producción y el consumo es un proceso entrópico. Este consiste en que altas concentraciones de energía son convertidas en artefactos útiles para la producción y el consumo, y durante esta etapa los artefactos son convertidos en desperdicio. La energía de la naturaleza (el sol, el viento...) causa que la materia se disperse; para volver a reconcentrar ese material disperso se requiere una cantidad muy importante de energía orientada a la regeneración de toda la materia que se ha utilizado, lo que hace que el proceso de reciclaje al cien por ciento sea prácticamente imposible. En consecuencia, tanto la materia como la energía son entrópicas.

La teoría neoschumpeteriana goza de un notable vigor intelectual, prueba de ello son las publicaciones y encuentros generados por la Society for Ecological Economics (Sociedad para la Economía Ecológica) y en el seno de la prestigiosa revista científica Evolutionary Economics (Economía Evolucionista). Desde esta organización se han promovido trabajos interesantes, como los modelos de sistemas ecológico-económicos o de la economía energética (a partir de los trabajos seminales de Costanza, 1997), que han sido particularmente influyentes en los siguientes campos: a) el impacto generado por el cambio tecnológico en las organizaciones, b) la capacidad de adaptación de las organizaciones en un entorno de cambio constante, c) la racionalidad limitada de los agentes, d) la forma en la que fluye la información, e) la generación del conocimiento y su eficiente y eficaz difusión, f) la variedad, novedad e indeterminación, g) la teoría del caos y la dinámica no-lineal, h) las relaciones entre individuos, grupos e instituciones y i) el papel del empresario en el desarrollo económico, entre otros.

\section{Los principios del pensamiento neoschumpeteriano y su utilidad para el estudio de la administración de empresas}

Para los neoschumpeterianos, las señales que la empresa recibe del sistema económico en el que opera son de tres tipos:

- Las oportunidades tecnológicas y los beneficios esperados asociados a ellas, tanto en sus propios productos como en otros.

- El comportamiento actual y esperado de la demanda por sus productos y otros productos.

- Los cambios en costos, precios, cantidades y rentabilidades en los mercados en los que opera y en algunos otros.

El primer grupo de señales corresponden a las oportunidades de innovación/imitación/mejora tecnológica, o estrategia de ajuste schumpeteriana. El segundo grupo de señales se relaciona con la búsqueda de oportunidades de crecimiento, o estrategia de ajuste keynesiana. El tercer grupo de señales se refiere a los ajustes de precio y/o cantidad con tecnología constante, o estrategia neoclásica de ajuste. En todo momento debe recordarse que la dirección y grado de los procesos de ajuste microeconómicos están determinados por las políticas, reglas 
sociales implícitas, formas dominantes de organización y coordinación entre los agentes económicos, y el grado y forma de los conflictos industriales y laborales.

El pensamiento neoschumpeteriano ha adoptado de la teoría poskeynesiana diversos elementos relacionados con el análisis específico de las empresas y su relación con el mercado. Una muestra de ello es que ambos enfoques comparten su rechazo a tres axiomas, considerados irrefutables por la teoría neoclásica: la neutralidad del dinero, la fácil sustitución en el consumo y en la producción y la predecibilidad del futuro (véase Dow, 2001). En virtud de ello, la compatibilidad entre los dos enfoques gravita en torno a que:

- La tasa de inversión es la variable que determina el crecimiento económico y la distribución del ingreso.

- El efecto ingreso supera al efecto sustitución.

- En el largo plazo las fuerzas del mercado no siempre son suficientes para conducir a un crecimiento económico.

- Las instituciones monetarias y crediticias desempeñan un papel fundamental en el sistema económico. Para el efecto, es fundamental la flexibilidad del sistema bancario, porque: a) de ello dependerá su capacidad para responder a la demanda que las empresas ejercerán en busca de crédito y b) el banco central podrá intervenir para sostener la liquidez y la confianza.

- Se reconoce el poder y la influencia que ejercen las empresas transnacionales, y el hecho de que, en muchos casos, gobiernos y sindicatos están subordinados a estas.

- Los precios y los salarios están administrados por el mercado, lo que significa que las decisiones de los usuarios y consumidores no inciden sobre los precios.

- No es factible controlar los procesos inflacionarios mediante instrumentos de política monetaria porque esta última es resultado de un conflicto sobre la distribución del ingreso; razón por la cual es indispensable una política de ingresos.

- Los mercados no son competitivos en absoluto, lo que significa que la distribución del ingreso no se realiza a través de los diferentes mecanismos que impone el mercado.

- Se da por sentada la aceptación de la existencia de la incertidumbre (en lugar del riesgo).

- El hombre es un ser social que goza de racionalidad limitada y que busca alcanzar metas (contrario a los planteamientos de los neoclásicos, que dan por hecho que el hombre es un ente solitario, racional y plenamente maximizador de recursos).

- Las leyes de la oferta y la demanda son interdependientes.

- El dinero es una medida de valor, el cual permite reducir la incertidumbre (los autores neoclásicos lo consideran sólo un medio de cambio).

Estas propuestas resultan particularmente sugerentes para las investigaciones sobre los fundamentos macroeconómicos del comportamiento microeconómico, debido a que se da por descontado que existe una influencia interdependiente entre ambos niveles de actividad. Esta cuestión permite argumentar la pertinente participación del Estado a través de la creación de instituciones y organizaciones fuertes y la selectiva y limitada regulación de las empresas privadas, todo lo cual permitirá 
atemperar la incertidumbre. La presencia del ente gubernamental debe ser inmanente en aquellos sectores considerados neurálgicos, como el de la educación y la generación de conocimiento, la sanidad, la creación de infraestructuras productivas y de transporte, ello con la esperanza de que a la postre se generen suficientes incentivos para una activa participación de la inversión privada. Asimismo, los neoschumpeterianos consideran que la economía es un complejo cúmulo de proposiciones enmarcadas dentro de los parámetros del denominado realismo crítico (tan explorado por autores como Lee, 2002: 790), y que apuntan a cuatro postulados básicos:

- La economía no solo se alimenta de eventos, además se nutre de estructuras y mecanismos causales que, en general, son plenamente identificables.

- Las estructuras y los mecanismos causales existen con independencia de que los sujetos los reconozcan e identifiquen.

- Los eventos económicos son ocasionados por un conjunto de estructuras y mecanismos causales.

- Los hechos están constituidos por: eventos empíricos, eventos que subyacen a los eventos empíricos y mecanismos y estructuras causales. En este contexto, la labor del economista es la de identificar las estructuras y mecanismos causales y, de ese modo, poder describir su influencia y acción sobre eventos concretos.

Para los neoschumpeterianos, estas cuatro proposiciones conducen a que el análisis económico debe realizarse en términos del tiempo histórico. Como lo dedujo Dow (2001: 13), los sistemas sociales se conciben como complejos, estructurados $\mathrm{y}$ transformables, por lo cual es necesario que sean analizados desde todos los ángulos posibles. Y respecto del mercado, los neoschumpeterianos consideran que, con independencia de su taxonomía, en este prevalece un entorno caracterizado porque el flujo de la información sobre la demanda es costoso e incierto, la comunicación entre las empresas, los proveedores, los clientes, las otras empresas rivales y las autoridades gubernamentales tiene lugar en un ambiente de incertidumbre, gracias a lo cual las empresas están imposibilitadas de ser agentes maximizadores o minimizadores (como lo ha estudiado Lavoie, 2001: 21). En este entorno incierto los empresarios y/o los consejos de administración están obligados a asumir decisiones, en la medida de lo posible y lo deseable, bajo la consideración de que estén basadas en datos que les permitan rastrear y evaluar los resultados financieros y no financieros.

Asimismo, este acervo de teorías considera que la principal función de las empresas es orientarse a encontrar lo que actualmente está oculto o se desconoce, y no, como creen los neoclásicos, hacer el mejor uso de lo que se conoce. Por ello, las empresas son organizaciones en las que prevalece la coordinación de esfuerzos, de la cual podrán generarse nuevos elementos que permitan elegir las decisiones más acertadas. Este principio es definitivo, porque en él reposa el interés de las empresas en favorecer el aprendizaje interactivo y cooperativo entre las personas que trabajan en ellas. De la adecuada coordinación depende, además de la generación de nuevo conocimiento y nueva información, la posibilidad para el lanzamiento de nuevos productos al mercado. Del mismo modo, la compenetración entre los empleados también es importante porque genera códigos de lenguaje y comunicación. Por tanto, el trabajador es considerado uno de los 
elementos de la cadena de producción, de ahí su enorme relevancia y la necesidad de hacer que desarrolle una alta fidelidad a la empresa. El despido de un trabajador implica la ruptura de rutinas y conocimiento $\mathrm{y}$, por tanto, representa un costo para la empresa.

Estos elementos conducen a considerar que las empresas son la mejor demostración de los límites del mercado; de hecho, en cierto modo son las empresas las encargadas de reemplazar al mercado con sus jerarquías, rutinas, reglas y controles que usualmente imponen, tanto explícita como tácitamente. En consecuencia, las empresas son un medio de coordinación desde las que se generan decisiones estratégicas, operaciones de mercado con el entorno, y jerárquicas al interior de las mismas, lo que se traduce en mecanismos de distribución del poder. Las estrategias son las elecciones que asumen los empresarios y los administradores con el interés de reducir la incertidumbre mediante la adquisición de mayor control sobre más recursos y procesos, lo que conduce al crecimiento de las empresas (Dunn, 2002: 68). Y sobre la cuestión del poder, las empresas lo buscan porque en la medida en que lo tengan podrán ejercer un control sobre sus proveedores, sus clientes, el gobierno y el uso de la tecnología. En este contexto, el dinero es una expresión de las relaciones de poder entre las empresas y la presencia de incertidumbre en el entorno.

Los neoschumpeterianos señalan que los precios desempeñan tres funciones principales: a) de posicionamiento (por ejemplo, los precios relativos entre bienes sustitutos), b) estratégicas (como las estrategias competitivas adoptadas por las empresas) y c) financieras (como la recolección de fondos internos para el mantenimiento de las tasas de inversión).
En este sentido, en las empresas que fijan sus precios en base al costo medio más un margen, los costos medios variables y marginales suelen ser constantes sobre amplios rangos de producción, al tiempo que los costos medios totales son decrecientes. Con fundamento en lo anterior, algunas de las características de los precios son: a) no indican escasez o abundancia, sólo reflejan los costos, b) se fijan en función de los que se consideran costos normales y se les añade un margen, c) se fijan en consideración a las transacciones repetidas, d) no son capaces de equilibrar el mercado y e) muchas veces son fijados antes de que se conozca el precio de los insumos. Estos son algunos de los supuestos y teorías del nutrido elenco de los que se alimenta el enfoque neoschumpeteriano.

\section{Conclusiones}

Los principios del pensamiento neoschumpeteriano se centran en el uso de los conceptos de la evolución biológica para analizar el comportamiento de las organizaciones y los sistemas económicos. Defienden que las posibilidades de progreso del sistema económico dependen fundamentalmente de su capacidad para innovar y no tanto de la esperanza de alcanzar un estado estacionario. Para este enfoque, desarrollo y crecimiento son en realidad dos procesos diferentes: el crecimiento se orienta a una trayectoria de insostenibilidad, mientras que el desarrollo se interesa en ser sostenible. Sin embargo, el desarrollo no es posible si antes no se produce un proceso de crecimiento. Es gracias a la dinámica que se genera en los procesos de cambio acumulativo (típicos del crecimiento económico) que son posibles las transformaciones radicales (típicas del desarrollo económico). 
Asimismo, los hábitos y las rutinas en el desarrollo económico juegan un papel parecido al de los genes en la evolución biológica (y también en el desarrollo de las organizaciones); es decir, son los depositarios del conocimiento acumulado en el tiempo (herencia) -y como tal, elementos de estabilidad-, pero simultáneamente los cambios en ellos permiten que las organizaciones progresen y se desarrollen (mutación). Y son los empresarios y/o los consejos de administración los encargados de su modificación: en su afán por incrementar ganancias modifican rutinas, y al hacerlo crean nuevas trayectorias de evolución, para las cuales hay que crear nuevos hábitos y nuevas instituciones. En el caso de las grandes empresas, las decisiones se asumen por los consejos de administración u otros cuerpos colegiados, quienes son los responsables de generar nuevas trayectorias de evolución. La noción del empresario, como tomador único e infalible de decisiones, es un enfoque obsoleto y que no tiene aplicación en el seno de las grandes empresas.

La teoría neoschumpeteriana es útil para el estudio de la administración de empresas en torno a aspectos como: a) la tasa de inversión es la variable que determina el crecimiento de las empresas, b) el efecto ingreso supera al efecto sustitución, c) en el largo plazo las fuerzas del mercado no siempre son suficientes para conducir a un crecimiento de las empresas, d) las instituciones monetarias y crediticias desempeñan un papel fundamental en el desarrollo de las empresas, e) se reconoce la existencia del poder y la influencia que ejercen las empresas transnacionales y que, en muchos casos, los gobiernos y los sindicatos están subordinados a estas, f) los precios y los salarios están administrados por el mercado, lo que significa que las decisiones de los usuarios y los consumidores no inciden sobre los precios, g) no es factible controlar los procesos inflacionarios mediante instrumentos de política monetaria porque esta es resultado de un conflicto sobre la distribución del ingreso, h) los mercados no son competitivos en absoluto, i) el hombre es un ser social que goza de racionalidad limitada y que busca alcanzar metas j) las leyes de la oferta y la demanda son interdependientes y k) el dinero es una medida de valor, el cual permite reducir la incertidumbre, entre otros factores. 


\section{Referencias bibliográficas}

BECKER, G. S. 1976. Altruism, Egoism and Genetic Fitness: Economics and Sociobiology. The Journal of Economic Literature. September, vol. 14, n. ${ }^{\circ}$ 3, págs. 817-826.

BOULDING, K. 1974. Evolutionary Economics. London: Routledge.

1983. Technology in the Evolutionary Process. En: S. McDonald y T. Mc Lamberton. The Trouble with Technology. New York: St. Martin's Press.

CLARK, C. 1967. Las condiciones del progreso económico. Madrid: Alianza.

CLARK, N. y JUMA, C. 1987. Long-run Economics: an Evolutionary Approach to Economic Growth. London: Frances Printer.

COSTANZA, R. (ed.). 1997. An introduction to Ecological Economics. Chicago: CRC Press.

DALY, H. E. 1968. On Economics as a Life Science. Journal of Polytical Economy. May-June, vol. 76, págs. 392-406.

1974. The Economics of the Steady-State. American Economic Review. May, vol. 64, n. ${ }^{\circ} 2$, págs. 15-21.

DENNETT, D. C. 2004. Freedom Evolves. London: Penguin Group.

DOSI, G. 1988. Technical Change and Economic Theory. London: Pinter Publishers.

1990. The Economics of Technical Change and International Trade. London: Pinter Publishers.

DOSI, G. y KOGUT, B. 1993. National Specifities and the Context of Change: The Coevolution of Organization and Technology. En: B. Kogut. Country Competitiveness. London: Oxford University Press.
DOSI, G.; PAVITT, K. y SOETE, L. 1990. The Economics of Technical Change and International Trade. London: Pinter Publishers.

DOW, S. 2001. Post Keynesian Methodology. En R. Holt y S. Presuman. A New Guide to Post Keynesian Economics. London: Routledge.

DUNN, S. 2002. A Post Keynesian Approach to the Theory of the Firm. En: S. Dow y J. Hilliard. Post Keynesian Econometrics, Microeconomics and the Theory of the Firm. London: Edward Elgar.

ENGLAND, R. W. 1994. Evolutionary Concepts in Contemporary Economics. Ann Arbor: University of Michigan.

ESSER, K. et al. 1996. Systemic Competitiveness: New Governenace Patterns for Industrial Development. London: Frank Cass.

FREEMAN, C. y PÉREZ, C. 1988. Structural Crises of Adjustment, Business Cycles and Investment Behaviour. London: Pinter Publishers.

FREEMAN, C. y SOETE, L. 1997. The Economics of Industrial Innovation. Boston Mass: The MIT Press.

FRIEDMAN, M. 1953. Essays in Positive Economics. Chicago: University of Chicago Press.

GEORGESCU-ROEGEN, N. 1967. Analytical Economics: Issues and Problems. Cambridge Mass: Harvard University Press.

(1971). 1996. La ley de la entropía y el proceso económico. Madrid: Fundación Argentaria.

- 1978. Los modelos dinámicos y el crecimiento económico. México: Lecturas del Trimestre Económico. 
HODGSON, G. 1995. Economía y evolución. Madrid: Celeste.

1996. Economics and Biology. London: Routledge.

2004. The Evolution of Institutional Economics: Agency, Structure and Darwinism in American Institutionalism. London: Routledge.

JIMÉNEZ HERRERO, L. M. 1996. Desarrollo sostenible y economía ecológica. Madrid: Síntesis.

LAVOIE, M. 2001. Pricing. En R. Holt y S. Pressman. A New Guide to Post Keynesian Economics. London: Routledge.

LEE, F. 2002. Theory Creation and the Methodological Foundations of Post Keynesian Economics. Cambridge Journal of Economics. $\mathrm{N}^{\circ}{ }^{\circ}$ 26, págs. $790-803$.

LEYDESDORFF, H. y ETZKOWITZ, H. (Eds.). 1997. A triple Helix of UniversityIndustry-Government Relations. The Future Location of Research, Book of Abstracts. New York: Science Policy Institute / State University of New York.

1996. Emergence of a Triple Helix of University-Industry-Government Relations. New York: Science and Public Policy / State University of New York.

LEYLESDORFF, L. y BESSLAAR, P. (Eds.). 1994. Evolutionary Economics and Chaos Theory: New Directions in Technology Studies. London: Pinter.

LOASBY, B. J. 1991. Equilibrium and Evolution: An Exploration of Connecting Principles in Economics. Manchester: Manchester University Press.

MARSHALL, A. 1919. Industry and Trade: A Study of Industrial Technique and Business Organization and their Influences on the
Conditions of Various Classes and Nations. London: MacMillan.

NELSON, R. 1993. National Innovation Systems: A Comparative Study. New York: Oxford University Press.

NELSON, R. y WINTER, S. 1982. An Evolutionary Theory Of Economic Change. Cambridge Mass: Harvard University Press.

ROSCHER, W. (1843). 1981. Outline of Lectures on Political Economy According to the Historical Method. Cambridge UK.: Cambridge University Press.

(1894). 1981. System of Political Economy. Cambridge UK: Cambridge University Press.

SCHMOLLER, G. (1900). 1993. Grundriss der allgemeinen Volkswirtschaftslehre. Berlin: Berlin Frein Universitat.

SCHUMPETER, J. A. 1911. The Theory of Economic Devlopment. Cambridge Mass: Harvard University Press.

SZENBERG, M. (Ed.). 1992. Eminent Economists. Cambridge Mass: Cambridge University Press.

VEBLEN, T. B. 1919. The Place of Science in Modern Civilisation. Chicago: Chicago University Press.

VERSPAGEN, B. 1993. Uneven Growth Between Interdependent Economies: The Evolutionary Dynamics of Growth and Technology. Avebury: Aldershot.

VROMEN, J. J. 1995. Economic Evolution: An Enquiry into the Foundations of New Institutional Economics. London: Routledge.

WINTER, H. 2005. Trade-offs. An Introduction to Economic Reasoning and Social Issues. Chicago: The University of Chicago Press. 